\title{
Individual differences in working memory capacity and visual search: The roles of top-down and bottom-up processing
}

\author{
KENITH V. SOBEL \\ University of Central Arkansas, Conway, Arkansas \\ MatThew P. GerRie \\ Victoria University of Wellington, Wellington, New Zealand \\ AND \\ Bradley J. Poole and Michael J. Kane \\ University of North Carolina, Greensboro, North Carolina
}

\begin{abstract}
Individual differences in working memory capacity (WMC) have been implicated in a variety of top-down, attention-control tasks: Higher WMC subjects better ignore irrelevant distractions and withhold habitual responses than do lower WMC subjects. Kane, Poole, Tuholski, and Engle (2006) recently attempted to extend these findings to visual search, but found no relation between WMC and search efficiency, even in difficult tasks yielding steep search slopes. Here we used a visual search task that isolated the contributions of top-down versus bottom-up mechanisms, and induced a habitual response via expectation. Searches that relied primarily on bottom-up mechanisms did not vary with WMC, but searches that relied primarily on top-down mechanisms showed an advantage for higher over lower WMC subjects.
\end{abstract}

People are equipped with an alliance of attentional mechanisms that sort through the constant flood of sensory input in order to select a few streams to process more thoroughly than the rest. Bottom-up mechanisms automatically and involuntarily select objects that stand out from the crowd, presumably because such novel objects are likeliest to require immediate consideration. Top-down mechanisms, in contrast, draw on our knowledge to select objects most likely to help us realize our longer-term goals (Connor, Egeth, \& Yantis, 2004). These two sorts of mechanisms often act at cross purposes: Top-down mechanisms underlie our ability to control the focus of attention, thereby ignoring conspicuous distractors that strongly activate, or capture, bottom-up mechanisms.

Insofar as top-down mechanisms facilitate the controlled deployment of attention, one might reasonably expect them to conspire with the "central executive" processes described in Baddeley and Hitch's (1974) model of working memory. Indeed, recent work has shown that measures of working memory capacity (WMC) correlate well with the attention-control ability to restrain a habitual response to a salient stimulus. For example, the abrupt onset of a peripheral light typically elicits an orienting saccade. When instructed to counter such automatic saccades with the opposite response, higher WMC subjects can more effectively execute "antisaccades" away from such abrupt onsets (Kane, Bleckley, Conway, \& Engle, 2001; Unsworth, Schrock, \& Engle, 2004). Similarly, people with higher WMC are better able to avoid Stroop interference (Kane \& Engle, 2003; Kiefer, Ahlegian, \& Spitzer, 2005; Long \& Prat, 2002) and to ignore conspicuous words spoken in the unattended channel during dichotic listening (Conway, Cowan, \& Bunting, 2001) than are people with lower WMC.

Top-down activation is also commonly presumed to affect the efficiency of visual search for a single target object embedded in a display containing several nontarget distractors, as indicated by several models of visual search (Cave, Kim, Bichot, \& Sobel, 2005; Wolfe, 1994). Moreover, experimental manipulation of WMC, via dual-task procedures, shows that visual search suffers when WMC is occupied (e.g., Allen, Baddeley, \& Hitch, 2006; Han \& Kim, 2004; Woodman \& Luck, 2004). In this context, Kane, Poole, Tuholski, and Engle (2006) designed three experiments to test the correlation between individual differences in WMC and visual search efficiency. They found that, across several varieties of search tasks in each experiment, including feature-absence search, conjunction search, and spatial-configuration search, all of which yielded steep search slopes, WMC was unrelated to search

K.V.Sobel, k.sobel@mac.com 

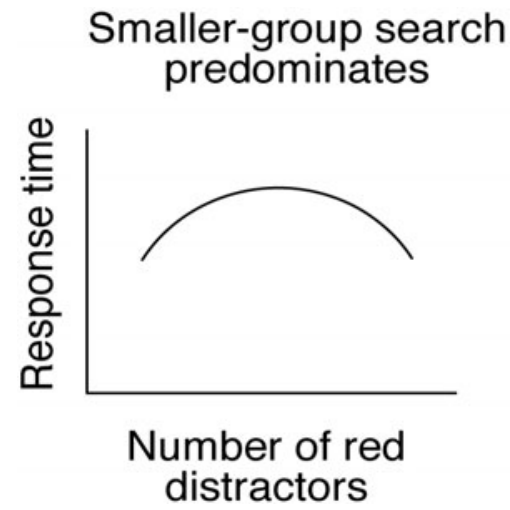

Target-color search predominates

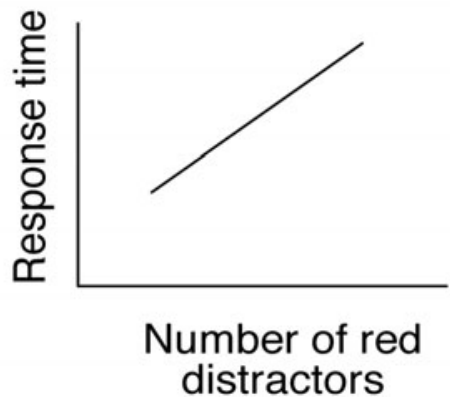

Figure 1. Hypothetical response time curves resulting from visual searches in which display size is held constant but the relative contribution of two types of distractor is varied. An inverted-U pattern indicates search through the smaller group; monotonic increase indicates target-color search.

efficiency. Kane et al. (2006) recognized, however, that WMC-sensitive control of search might be more likely in other experimental contexts, for example in those that demand the constraint of visual focus away from consistent distractor locations or in those that encourage top-down modulation of features that are likely to distinguish the search target. Here we explored the latter type of search task.

\section{A NEW VISUAL SEARCH TASK}

One of the tasks investigated by Kane et al. (2006) was the venerable conjunction search, in which subjects searched for a red vertical line among displays containing varying numbers of distractors. Some of the distractors had the same color as the target but a different orientation (hereafter, color items), and others had the same orientation as the target but a different color (hereafter, orientation items). The target appeared in half of the trials, and subjects indicated whether it was present. The number of distractors (display size) was manipulated by adding equal numbers of both distractor types. As in many conjunction search experiments, response time (RT) increased monotonically with display size. However, search slopes did not vary with WMC. We modified Kane et al.'s (2006) method in two important ways.

\section{Manipulating Distractor Orientations}

Do subjects search through the entire conjunctionsearch display, or do they restrict search to a single feature-defined subset? Egeth, Virzi, and Garbart (1984; see also Friedman-Hill \& Wolfe, 1995) noted that in experiments in which display size is increased by adding equal numbers of two types of distractor, any variations in the overall display size are perfectly confounded with variations in the size of each set of distractors. As a result, in traditional conjunction searches it is impossible to decide whether increasing RT functions indicate search through the entire display, or through just one set of distractors. To eliminate this confound Egeth et al. decoupled the set sizes of the two distractor types; later researchers extended this idea by holding the overall display size constant and varying the relative contribution of the two distractor types (Poisson \& Wilkinson, 1992; Shen, Reingold, \& Pomplun, 2000; Sobel \& Cave, 2002; Zohary \& Hochstein, 1989). Thus, in some displays the color items outnumber the orientation items, in some displays the distractor types are equally represented, and in some displays orientation items outnumber color items.

Bacon \& Egeth (1997) argued that in conjunction searches of this sort, the shape of the resulting RT curve reveals the range of objects to which search is restricted, as depicted in Figure 1. If search is restricted to the smaller group, RTs should be short when the color group is much smaller than the orientation group, rising to a maximum when the two groups are equal in size, then falling again to the point at which the orientation group is much smaller than the color group. In contrast, if search is restricted to the color group, regardless of its relative size, RTs should increase linearly with the size of the color group.

Sobel and Cave (2002) manipulated distractors' orientations so that they were either very distinct from, or very similar to, the target. In the distinct-orientations condition, subjects searched through the smaller group, whichever it happened to be (color or orientation). In the similarorientations condition, however, subjects restricted search to the target-color group, regardless of its size, as if targetorientation were a difficult cue to use.

When searching through displays consisting of groups of unequal sizes, each member of a smaller group tends to be more distinct and thus salient from other display items than each member of a larger group; Sobel and Cave (2002) thus argued that smaller-group search is driven primarily by the activation of bottom-up mechanisms. On the other hand, the members of a feature-defined group do not enjoy a benefit of salience when that group is the larger group, so restricting search to a feature-defined subset must rely primarily on a top-down grouping strategy (see also Kaptein, Theeuwes, \& van der Heijden, 1995). Here, then, is the first of two principles guiding our current task 
design: manipulating the discriminability of the target's and distractors' orientations allows us to selectively vary the relative contributions of bottom-up and top-down mechanisms. If, as in other attention tasks, WMC correlates better with "top-down" processing than "bottom-up," then WMC should affect performance primarily on search displays with similar orientations.

\section{Inducing a Habitual Response}

To further increase WMC contributions to search, we induced a habitual response as in Kane and Engle (2003), who tested for WMC differences in the Stroop task. In some trials the color word and its hue matched (congruent trials) while in other trials they did not (incongruent trials). When the task presented very few congruent trials, Stroop interference had only a weak association to WMC, but when congruent trials greatly outnumbered incongruent trials, low WMC subjects committed many more wordreading errors than did high WMC subjects. Apparently, the abundance of congruent trials reinforced the habitual response of reading the color word rather than naming the hue, but higher WMC subjects were better able to override this habit. We considered the distinct-orientations condition here to be analogous to the congruent trials in Kane and Engle, and the similar-orientations condition to be analogous to the incongruent trials. That is, we presumed performance in the distinct-orientations and congruent trials to be driven primarily by bottom-up factors; in the similar-orientations and incongruent trials to be driven more by top-down factors. Taking a cue from Kane and Engle, we designed the distinct-orientations condition to induce a habitual response to preferentially search through the group with the target's color. How did we do so? In one condition of a conjunction search in Bacon and Egeth (1997), the color group was most often smaller than the orientation group. Skewing the trials in this way meant that restricting search to the color group was generally extremely efficient, thus engendering a general strategy of searching through the color group.

\section{Predictions}

In the present task, we combined these two influences, manipulating the distractors' orientation and thereby the bottom-up salience of the target's orientation, and skewing the trial distributions in the distinct-orientations condition to create the expectation that target-color search was generally advantageous. Consider the search for a red horizontal line. When one group of distractors (e.g., red verticals) is smaller than the other (e.g., green horizontals), both of the features that define the smaller group will receive bottom-up activation because these features stand out from the more common features of the larger group. Relatively modest levels of top-down control should be required to accord higher priority to the feature that these items share with the target (red) than to the feature that these items do not share with the target (vertical).

If both of the target's features are distinct from the distractors' features, the smaller group, whichever it is, will enjoy a bottom-up advantage. However, in the similar-orientations condition the bottom-up salience of the target's orientation is weak, thereby requiring much more precise top-down control to prioritize orientation (as compared to prioritizing color). Thus if WMC differences are related to the ability to exert top-down control over bottom-up information, then low WMC subjects should differ from high WMC subjects primarily when (1) orientation differences are small (thus making color differences more salient than orientation differences, and leading subjects to search by color even as the number of color distractors increases) and (2) the smaller distractor group is the one with a different color as the target (thus producing a bottom-up salience advantage for the nontarget color).

\section{METHOD}

\section{Subjects}

Forty undergraduates (ages 18-25 years) participated for course credit. All reported normal color vision.

\section{WMC Screening}

We developed an automated version of an operation span (OSPAN) task, based on Unsworth, Heitz, Schrock, and Engle (2005). Subjects used a computer mouse to interact with the program. Each trial presented a simple arithmetic problem (e.g., $2 \times 7+3=$ ), together with a button labeled "Continue." When subjects solved the problem, they clicked the mouse button to present the next display, which provided an answer (e.g., 17) and 2 buttons to click, labeled "True" and "False." After response, a letter appeared for $1 \mathrm{sec}$, followed by a 1 -sec blank screen.

Trials were organized into sets, each containing three to seven equation-word pairs. At the conclusion of each set, a window appeared with 12 buttons, each labeled with a letter. Subjects clicked the buttons to serially recall the set's letters. If all were recalled in serial order, the number of points equal to the number of items in the set was awarded. Next, a display indicated the number of correct arithmetic responses from the set and a running total percent correct. We urged subjects to improve if arithmetic performance dropped below $85 \%$. All subjects exceeded this overall criterion.

After three practice trials of set size 3, test trials comprised three repetitions of sets containing three, four, five, six, or seven trials, for a total of 75 possible points. Set order was determined randomly for each subject. After about half the trials, a display asked subjects to take a short break. Three practice trials followed the break.

\section{Visual Search}

Subjects viewed the computer screen from a distance of about $59 \mathrm{~cm}$. A centered fixation cross $\left(0.11^{\circ} \times 0.11^{\circ}\right)$ was visible throughout the experiment. All items in the search arrays were arranged on an imaginary, centered circle with a radius of $2.7^{\circ}$. The target, present on every trial, was a red horizontal arrow, $0.25^{\circ}$ long $\times 0.076^{\circ}$ wide, pointing either left or right. Distractors were either green horizontal and red vertical arrows (distinct orientations), or green horizontal arrows and red arrows rotated $20^{\circ}$ counterclockwise from horizontal (similar orientations). Subjects indicated the target's heading by pressing the right or left arrow key as quickly and accurately as possible. Following response, the array was erased for $1 \mathrm{sec}$, leaving only the fixation cross (and the word 'Incorrect' on error trials). After every 120 trials, a screen indicated the subject's progress and allowed a short break. The first three trials in the experiment and the first three trials following breaks were not analyzed.

Every search array contained one red horizontal target and 20 distractors but proportions of distractor types varied across trials. Distractor sets included 2, 6, 10,14, or 18 red distractors randomly intermixed with $18,14,10,6$, or 2 horizontal distractors. The target was assigned to one of four quadrants, and position within the quadrant was randomly determined. Thus, the factors constraining each search array were target heading (two levels), distractor orientation (distinct or similar ori- 


\section{Distinct orientations}
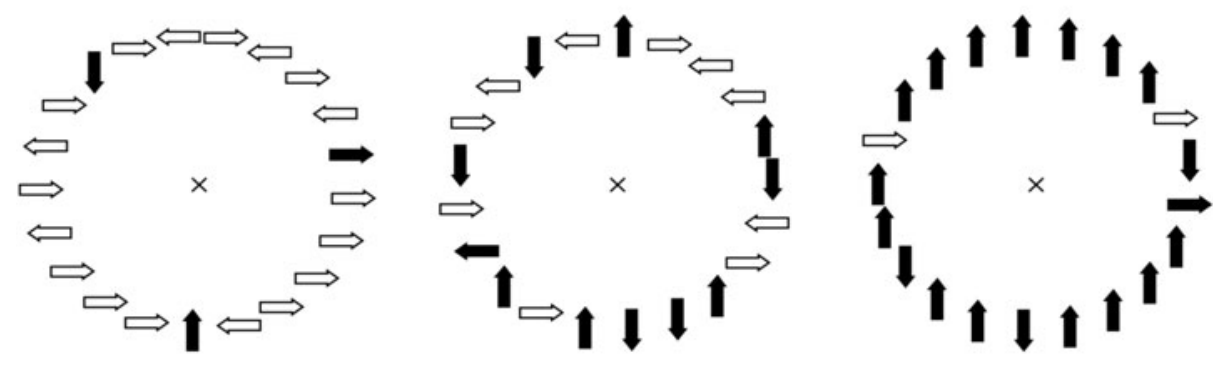

\section{Similar orientations}
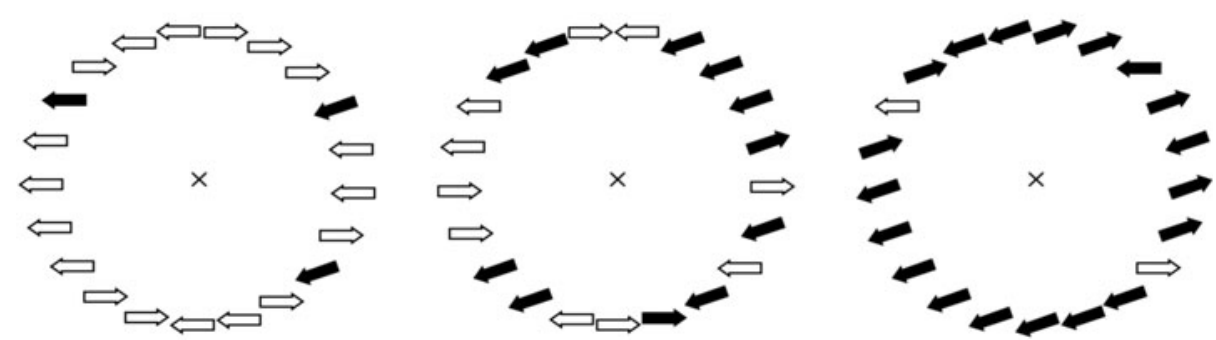

Figure 2. Three search arrays from the distinct-orientations condition (upper three displays), and three from the similar-orientations condition (lower three displays). Filled shapes indicate items that were colored red in the experimental display, empty shapes indicate items that were colored green. All search arrays contained a red horizontal arrow target along with 20 distractors. In some displays the target-color distractors predominated, in some the two distractor set sizes were roughly equal, and in some the target-orientation distractors predominated.

entations, two levels), distractor ratio (five levels) and target location (four levels); all were manipulated within subjects. Figure 2 depicts three search arrays from each of the distractor-orientation conditions.

The similar-orientations condition included 4 repetitions of every combination of target heading, distractor ratio and target location for 160 total trials. Because target heading and location were nuisance variables, there were effectively 32 trial repetitions for each level of distractor ratio in the similar-orientations condition. For the distinctorientations condition, trials in which the orientation group predominated were 3.5 times as numerous as those in which color group predominated (336 vs. 96 trials). Thus, the distinct-orientations condition presented 168 trials with 2 red distractors $(21$ repetitions of every combination of target heading and location, $21 \times 2 \times 4$ ), 168 trials with 6 red distractors, 32 trials with 10 red distractors, 32 trials with 14 red distractors, and 32 trials with 18 red distractors. Trial order was determined randomly for each subject.

\section{RESULTS}

High and low WMC groups (high-span subjects and low-span subjects) represented the top and bottom quartiles of OSPAN scores (for low-span subjects, $N=$ $10, M$ score $=18.0$, range $=4-27$; for high-span subjects, $N=10, M$ score $=62.1$, range $=48-75)$. We submitted visual search error rates to a three-way ANOVA with span group as a between-subjects variable and distractor orientation and distractor ratio as within-subjects variables. No main effects or interactions were significant.

Figure 3 depicts RTs as a function of distractor ratio and span group in both distractor-orientation conditions. A three-way ANOVA indicated that all main effects and interactions were significant. Low-span subjects responded more slowly than high-span subjects $[F(1,18)=13.6$, $p<.01]$; distinct orientations yielded shorter RTs than did similar orientations $[F(1,18)=199.3, p<.01]$; RTs varied across levels of distractor ratio $[F(4,72)=82.8$, $p<.01]$; and, RT slopes were steeper over distractor ratios in the similar-orientations than in the distinct-orientations condition $[F(4,72)=65.6, p<.01]$. Significant interactions of span group $\times$ distractor orientation $[F(1,18)=$ $7.1, p<.05]$, and span group $\times$ distractor ratio $[F(4,72)=$ $7.6, p<.01]$, were qualified by their three-way interaction $[F(4,72)=6.8, p<.01]$, indicating that RT slopes were unrelated to WMC for distinct orientations, but low-span subjects' slopes were steeper than high-span subjects' for similar orientations.

This three-way interaction supports the hypothesis that WMC should affect RT more in the similar-orientations than in the distinct-orientations condition. That is, bottom-up mechanisms provide good guidance in the distinctorientations condition, and so low-span subjects should perform no differently from high-span subjects. Predictions were more complicated for the similar-orientations results. Remember that in the distinct-orientations condition there were many more trials in which targetorientation distractors predominated, thereby reinforcing a habit to search through the color group throughout the experiment. In the similar-orientations condition, search 
through the color group is efficient when there are few target-color items, but this strategy becomes increasingly inefficient as the number of target-color items increases. Because we expected low-span subjects to be less able to override habitual target-color search than high-span subjects, low-span subjects should be relatively less efficient as the number of target-color items increased; in other words, low-span subjects' RT slopes should be steeper than high-span subjects'.

We used planned contrasts to test for such span differences in the linear and quadratic trends for the distinct- and similar-orientations conditions. For distinct orientations, neither contrast was significant $(F \mathbf{s}<1)$. For similar orientations, however, RTs were steeper for low than for high-span subjects $[F(1,144)=40.11, p<.01]$, and low-span subjects showed a more upward-deflecting quadratic trend in contrast to the high-span subjects' more downward-deflecting trend $[F(1,144)=10.52, p<.01]$. Although it may appear that the observed WMC differences are driven primarily by the 18-red-distractors condition, pairwise comparisons indicate that low-span subjects were slower than high-span subjects not just in the 18red-distractors condition $[F(1,72)=71.53, p<.01]$, but in the 10- and 14-red distractor conditions, as well ( $F \mathrm{~s}>$ $5.35, p \mathrm{~s}<.05)$.

\section{DISCUSSION}

Because WMC has been implicated in a variety of attention-control tasks, demonstrating its effects in conjunction search might seem like a formality. Nonetheless, Kane et al. (2006) showed this quest was not straightforward, and so in the present study we bore in mind that: (1) high-span subjects outperform low-span subjects on tasks that rely more on top-down than bottom-up processes (Kane et al., 2001), and (2) high-span subjects are better able to override habitual responses based on expectation than are low-span subjects (Kane \& Engle, 2003). To exploit these characteristics of WMC, we developed a conjunction search task that isolated top-down from bottom-up factors (e.g., Sobel \& Cave, 2002) and created the expectation that searching through the targetcolor group was more efficient than searching through the orientation group (e.g., Bacon \& Egeth, 1997).

The results supported our hypotheses. In the distinctorientations condition, both features of the smaller group received stronger bottom-up activation than the features of the larger group, and top-down processes were needed only to assign higher priority to whichever feature the smaller group shared with the target. With a relatively minor role for top-down factors, high- and low-span subjects performed equivalently. Moreover, in the similarorientations condition, when the group with the target's color was smaller, top-down control was required merely to give color higher priority than orientation; here again high- and low-span subjects performed equivalently. When green horizontals were the smaller group in the similarorientations condition, however, the bottom-up activation for orientation was substantially reduced, but was just as strong for color as in the distinct-orientations condition.
Here, substantial top-down control was required to override the misleading bottom-up guidance, giving rise to steeper RT slopes for low-span subjects than high-span subjects.

Why did Kane et al. (2006) find no WMC-related differences in conjunction search? Sobel and Cave (2002) argued that search in the distinct-orientations condition is guided primarily by bottom-up salience; by extension, most typical conjunction searches with two distinct target features (e.g., Kane et al., 2006) are guided by bottom-up salience as well. However, Sobel and Cave couched their descriptions of salience in terms of group size, with each member of the smaller group being more distinct from other items than each member of the larger group. How reasonable is it to extend their salience argument to tasks in which the two group sizes are identical? Note that studies that have manipulated distractor ratios have typically found slight
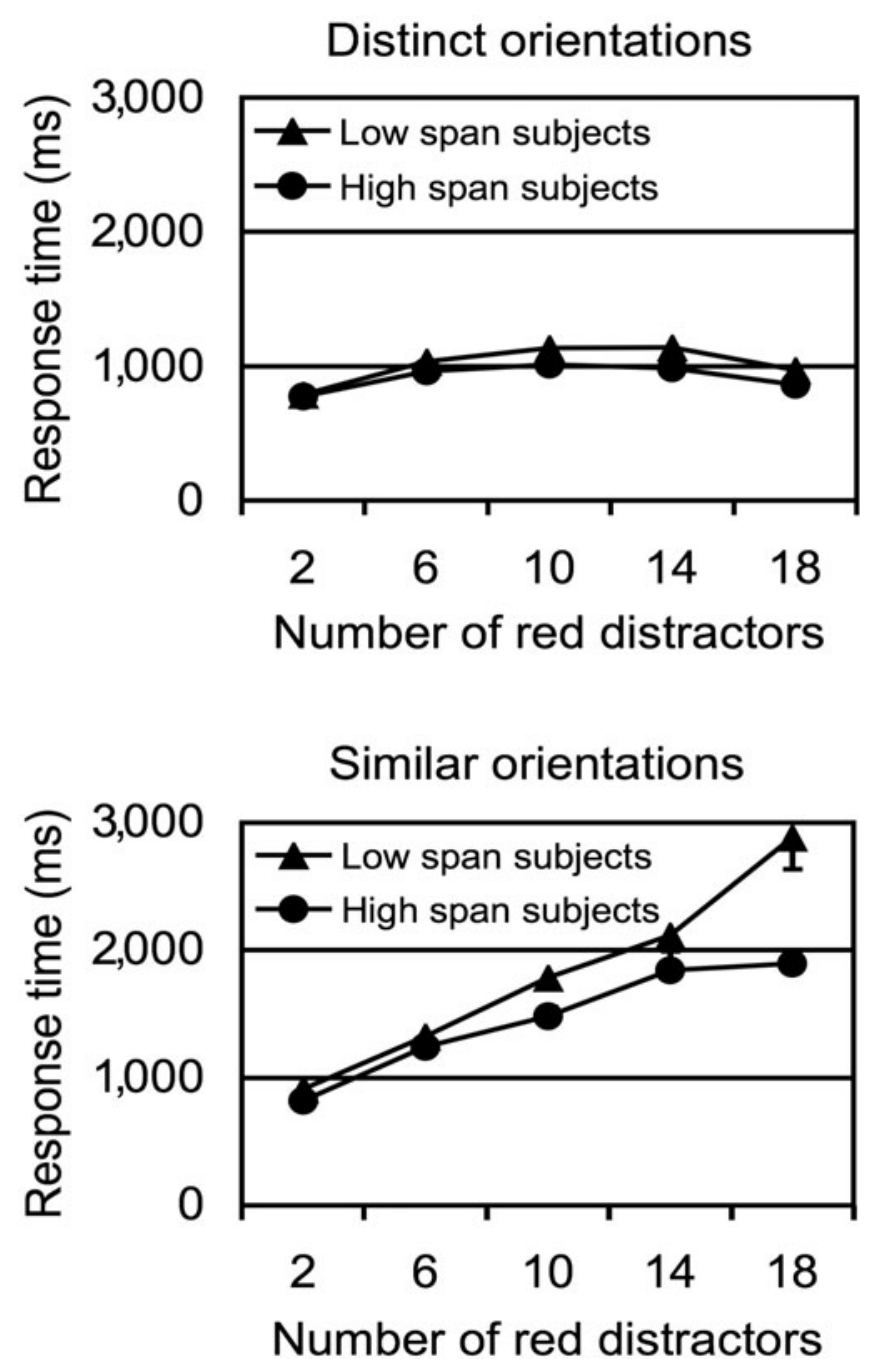

Figure 3. Response times in milliseconds from the visual search experiment plotted as a function of distractor ratio, for high-span and for low-span participants. Panel A depicts the data from the distinct-orientations condition, and Panel B depicts the data from the similar-orientations condition. Error bars represent standard error of the mean. 
asymmetries in their inverted-U RT patterns (e.g., Shen, Reingold, \& Pomplun, 2000; Zohary \& Hochstein, 1989), such that searches are more biased to proceed through color than orientation. Presumably this bias indicates that targetdistractor color differences are more salient than orientation differences. If so, then in the conjunction searches in Kane et al. (2006), although members of neither group enjoyed any salience advantage due to group size, members of the color group were nevertheless more salient, thereby recruiting bottom-up mechanisms to aid search.

\section{AUTHOR NOTE}

Correspondence concerning this article should be sent to K. V. Sobel, Department of Psychology and Counseling, University of Central Arkansas, 201 Donaghey Ave., Mashburn Hall 260, Conway, AR 72035 (e-mail:k.sobel@mac.com).

\section{REFERENCES}

Allen, R. J., Baddeley, A. D., \& Hitch, G. J. (2006). Is the binding of visual features in working memory resource-demanding? Journal of Experimental Psychology: General, 135, 298-313.

BACON, W. F., \& EGETH, H. E. (1997). Goal-directed guidance of attention: Evidence from conjunctive visual search. Journal of Experimental Psychology: Human Perception \& Performance, 23, 948-961.

BADDELEY, A. D., \& Hitch, T. (1974). Working memory. In G. H. Bower (Ed.), The psychology of learning and motivation (Vol. 8, pp. 47-89). New York: Academic Press.

Cave, K. R., Kim, M.-S., Вichot, N. P., \& Sobel, K. V. (2005). The FeatureGate model of visual selection. In L. Itti, G. Rees, \& J. Tsotsos (Eds.), Neurobiology of attention (pp. 547-552). New York: Elsevier.

Connor, C. E., Egeth, H. E., \& Yantis, S. (2004). Visual attention: Bottom-up versus top-down. Current Biology, 14, R850-R852.

Conway, A., Cowan, N., \& Bunting, M. (2001). The cocktail party phenomenon revisited: The importance of WM capacity. Psychonomic Bulletin \& Review, 8, 331-335.

Egeth, H. E., Virzi, R. A., \& Garbart, H. (1984). Search for conjunctively defined targets. Journal of Experimental Psychology: Human Perception \& Performance, 10, 32-39.

Friedman-Hill, S., \& Wolfe, J. M. (1995). Second-order parallel processing: Visual search for the odd item in a subset. Journal of Experimental Psychology: Human Perception \& Performance, 21, 531-551.

HAN, S.-H., \& KIM, M.-S. (2004). Visual search does not remain efficient when executive working memory is working. Psychological Science, 15, 623-628.
Kane, M. J., Bleckley, M., Conway, A., \& Engle, R. (2001). A controlled-attention view of WM capacity. Journal of Experimental Psychology: General, 128, 309-331.

KANE, M. J., \& ENGLE, R. (2003). Working-memory capacity and the control of attention: The contributions of goal neglect, response competition, and task set to Stroop interference. Journal of Experimental Psychology: General, 132, 47-70.

Kane, M. J., Poole, B. J., Tuholski, S. W., \& Engle, R. W. (2006). Working memory capacity and the top-down control of visual search: Exploring the boundaries of "executive attention." Journal of Experimental Psychology: Learning, Memory, \& Cognition, 32, 749-777.

Kaptein, N. A., Theeuwes, J., \& van der Heijden, A. H. C. (1995). Search for a conjunctively defined target can be selectively limited to a color-defined subset of elements. Journal of Experimental Psychology: Human Perception \& Performance, 21, 1053-1069.

Kiefer, M., Ahlegian, M., \& Spitzer, M. (2005). Working memory capacity, indirect semantic priming, and Stroop interference: Pattern of interindividual prefrontal performance differences in healthy volunteers. Neuropsychology, 19, 332-344.

LONG, D. L., \& PRAT, C. S. (2002). Working memory and Stroop interference: An individual differences investigation. Memory \& Cognition, 3, 294-301.

PoIsson, M. E., \& WiLKInson, F. (1992). Distractor ratio and grouping processes in visual conjunction search. Perception, 21, 21-38.

Shen, J., Reingold, E. M., \& Pomplun, M. (2000). Distractor ratio and grouping processes in visual conjunction search. Perception, 21, 21-38.

Sobel, K. V., \& CAVE, K. R. (2002). Roles of strategy and salience in conjunction search. Journal of Experimental Psychology: Human Perception \& Performance, 28, 1055-1070.

Unsworth, N., Heitz, R. P., Schrock, J. C., \& Engle, R. W. (2005). An automated version of the operation span task. Behavior Research Methods, 37, 498-505.

Unsworth, N., Schrock, J. C., \& Engle, R. W. (2004). Working memory capacity and the antisaccade task: Individual differences in voluntary saccade control. Journal of Experimental Psychology: Learning, Memory, \& Cognition, 30, 1302-1321.

Wolfe, J. M. (1994). Guided Search 2.0: A revised model of visual search. Psychonomic Bulletin \& Review, 1, 202-238.

Woodman, G. F., \& LucK, S. J. (2004). Visual search is slowed when visuospatial working memory is occupied. Psychonomic Bulletin \& Review, 11, 269-274.

ZOHARY, E., \& Hochstein, S. (1989). How serial is serial processing in vision? Perception, 18, 191-200.

(Manuscript received August 3, 2006; revision accepted for publication November 9, 2006.) 